\title{
LAS DIFICULTADES EN EL PROCESO ACADÉMICO SUSCEPTIBLES DE PRODUCIR RESILIENCIA, SEGÚN ALUMNOS DE ENFERMERÍA ESPAÑOLES
}

\author{
'Alvaro Bernalte Benazet ${ }^{2}$ María Teresa Miret García \\ ${ }^{3}$ Cristina María Navarro Carrasco ${ }^{3}$ Soledad Ruiz Martel \\ ${ }^{4}$ María Teresa Bermúdez Ferreiro ${ }^{1}$ María Mercedes Lupiani Jiménez \\ ${ }^{1}$ Dr. TEU ${ }^{2}$ Antropóloga Social ${ }^{3}$ Diplomada en Enfermería \\ ${ }^{4}$ Dra. en Pedagogía. Universidad de Cádiz
}

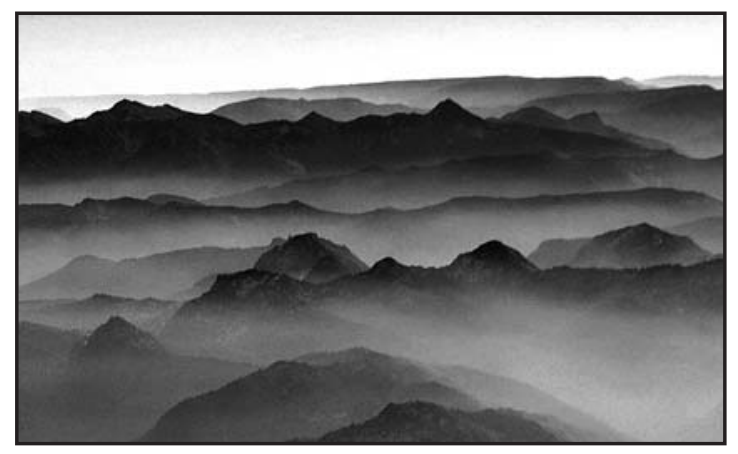

THE DIFFICULTIES IN THE ACADEMIC PROCESS CAPABLE OF PRODUCING RESILIENCIA, ACCORDING TO SPANISH UNIVERSITY STUDENTS OF NURSING

\section{ABSTRACT}

$\mathrm{T}$

The resiliencia is not a new concept, in fact the authors dedicated to his study, they admit the proliferation of articles about the topic and assume that it exists at par of the humanity. In the present study, we understand the resiliencia as the aptitude of the human beings to overcome adversities and to learn of it to continue with the daily life, the interest of the same one is that we have studied the adversities or difficulties that the pupils recognize like capable of producing resiliencia, instead of making it from the point of view discipline, of the professional, they will be analyzed from the point of view of the actors, in this case the student body of a University School of Nursing of Algeciras (Spain). The present work is a qualitative research, from the point of view cultural sight (least used in the study of the resiliencia), and we have used two associate technologies, namely: a Technology Delphi and one of nominal Group, later to confirm the obtained results, with the contributions with Brainstorming's Technology.

Between the results, it surprises that one that the pupils consider to be adversities or difficulties, and particularly the fact that the academic aspects that scarcely were supported as difficulties on having ended the Technology Delphi, they appear in the last technology, of contribution of ideas and therefore more creative, of forceful form.

Key words: Resiliencia; and Resiliencia in top education.

\section{AS DIFICULDADES NO PROCESSO ACADÊMICO SUSCEPTÍVEIS DE PRODU- ZIR RESILIÊNCIA, SEGUNDO ALUNOS DE ENFERMAGEM ESPANHÓIS}

\section{RESUMO}

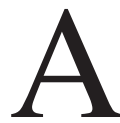

resiliência não é um conceito novo, mas de fato os autores dedicados ao seu estudo reconhecem a proliferação de artigos sobre o tema e afirmam que existe paralelamente com a humanidade. No presente estudo, entendemos a resiliência como a capacidade dos seres humanos de superar adversidades e aprender com essa situação para continuar com a vida cotidiana. $\mathrm{O}$ interesse do mesmo é que temos estudado as adversidades ou dificuldades que os alunos reconhecem como susceptíveis de produzir resiliência, em vez de fazê-lo desde o ponto de vista disciplinar, do profissional, serão analisadas desde o ponto de vista dos autores, neste caso, o alunado de uma 
Escola Universitária de Enfermagem de Algeciras (Espanha).

Este trabalho é uma pesquisa qualitativa, do ponto de vista cultural (o menos utilizado no estudo da resiliência), e utilizamos duas técnicas relacionadas, quais sejam: a técnica Delphi e de grupo nominal, para contrastar os resultados obtidos, com as contribuições de uma técnica de brainstorming.

Entre os resultados, surpreende o fato de que os estudantes consideram que as adversidades ou dificuldades, e especialmente o fato de que os aspectos acadêmicos que apenas se sustentavam como dificuldades, ao terminar a técnica Delphi, irromperam na última técnica, fornecendo ideias e, portanto, sendo mais criativa, de forma contundente.

Palavras-chave: Resiliência, Resiliência em Educação Superior.

\section{RESUMEN}

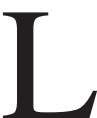

a resiliencia no es un concepto nuevo, de hecho los autores dedicados a su estudio, reconocen la proliferación de artículos sobre el tema y asumen que existe a la par de la humanidad. En el presente estudio, entendemos la resiliencia como la capacidad de los seres humanos de superar adversidades y aprender de ello para continuar con la vida cotidiana, el interés del mismo es que hemos estudiado las adversidades o dificultades que los alumnos reconocen como susceptibles de producir resiliencia, en vez de hacerlo desde el punto de vista disciplinar, del profesional, serán analizadas desde el punto de vista de los actores, en este caso el alumnado de una Escuela Universitaria de Enfermería de Algeciras (España).

El presente trabajo es una investigación cualitativa, desde el punto de vista cultural (el menos utilizado en el estudio de la resiliencia), y hemos utilizado dos técnicas asociadas, a saber: una Técnica Delphi y una de Grupo nominal, para posteriormente contrastar los resultados obtenidos, con las aportaciones con una Técnica de Brainstorming.

Entre los resultados, sorprende aquello que consideran los alumnos adversidades o dificultades, y particularmente el hecho de que los aspectos académicos que apenas se sostenían como dificultades al acabar la Técnica Delphi, irrumpen en la última técnica, de aportación de ideas y por tanto más creativa, de forma contundente.

Palabras clave: Resiliencia; y Resiliencia en educación superior

\section{INTRODUCCIÓN}

El término resiliencia proviene del latín resilio, que significa volver atrás, volver de un salto, resaltar y rebotar. Es un término utilizado en tecnología como la resistencia de un cuerpo a la rotura por un golpe, así un cuerpo es menos frágil cuanto más resiliente es.

La resiliencia pues, es una reacción ante un "golpe" lo que nosotros llamaríamos una dificultad o adversidad, que puede ser cultural en sentido amplio (económica, lenguaje, étnica...) como explicitan Tylor (1924) y Malinowski (1973), psicológica o física, que impulsa al que la padece a reaccionar ante ella superándola, pero con un matiz importante, que aprende de la experiencia pasada.

Según Rutter, (1993), la resiliencia es un concepto adaptado a las Ciencias Sociales para caracterizar a aquellas personas que, a pesar de nacer y vivir en situaciones de alto riesgo, se desarrollan sanos y exitosos es el término invulnerabilidad aplicado a niños que parecían constitucionalmente tan fuertes, que no cedían ante la presión del estrés y de la adversidad.

También podemos expresar la resiliencia decía Melillo, como la capacidad de los seres humanos de superar los efectos de una adversidad a la que están sometidos e, incluso, de salir fortalecidos de la situación (mencionado por Bermúdez, 2009).

Los autores dedicados a su estudio, reconocen la proliferación de artículos sobre el tema y asumen que existe a la par de la humanidad, Infante (2001) por ejemplo, describe la resiliencia en la historia de la infancia y sostiene que aunque sea un concepto reciente, su realidad es antigua. Este pensamiento es compartido también por autores como Manciaux M,; Vandistendael, S.; y cols. (2003), Cyrulnik, B. (2002), Vandistendael, S. (2003), Theis, A. (2003), y Saavedra, G. E. (2003) quien argumentó en este sentido, que el término se adap- 
tó de la ingeniería "en donde se utilizó para describir los materiales que recobraban su forma original, luego de sufrir las presiones de algún elemento externo".

El concepto de resiliencia se extiende cada vez más y su principal utilidad se ha registrado en países en desarrollo, si bien Suárez, O. N. (2001) atribuye su origen exclusivamente al hemisferio norte y señala que "a veces se le considera como un instrumento más de la dominación del norte sobre el sur y del mantenimiento silencioso de la inequidad”. En el año 2004, Suárez, O. N., afirmó que luego de desarrollarse el concepto en el hemisferio norte -con Rutter en Inglaterra y Werner en Estados Unidos- se extendió a toda Europa, Francia, Alemania y España, y finalmente llegó a América Latina.

El mismo Suárez, O. N. (2001:77-82) sostiene la existencia de tres corrientes: “... la norteamericana, esencialmente conductista, pragmática y centrada en lo individual; la europea, con mayores enfoques psicoanalíticos y una perspectiva ética; y la latinoamericana, de raigambre comunitaria, enfocada en lo social como lógica respuesta a los problemas del contexto". El creciente interés de las disciplinas por la investigación de la resiliencia, la convirtió según Suárez, O. N. (2004), en una actividad transdisciplinaria que agrupa los aportes de diversas áreas y sectores, de manera tal que "Múltiples saberes se asocian y comparten la generación del conocimiento sobre resiliencia y se fecundan en su aplicación práctica en proyectos sostenibles". Así tenemos según este autor, a la Psicología y sus aportes sobre el desarrollo psicosocial que sirvieron de base para el concepto; a la antropología, y a la sociología.

Existen multitud de conceptos relacionados con la resiliencia y aquí por motivos obvios, no vamos a realizar una revisión exhaustiva pero vamos a comentar algunos de ellos, por ejemplo el término personalidad resistente (hardiness), sobre esto Kobasa, Maddi y Kahn en sus obras desde los 70, refieren a ciertas personas que ante hechos vitales negativos parecían tener unas características de personalidad que les protegían.

Según Rutter (1985) la resistencia procede tanto del ambiente como de lo constitucional. Si bien en las primeras publicaciones alusivas a la resiliencia, se tendió a utilizar éste concepto como equivalente al de invulnerabilidad, hoy día ha quedado relegado al campo de la psicopatología.

Competencia es otro concepto que se maneja, así según Luthar (1993) los estudios de resiliencia se restringen (a veces) a la capacidad de competencia social, bajo el supuesto que ésta refleja buenas habilidades de enfrentamiento subyacentes, si bien a pesar de ello pueden ser vulnerables frente a problemas físicos o mentales.

Tomando en consideración los vacíos en el estudio de la resiliencia, se atiende a la importancia de descifrar la dinámica entre los niveles del marco ecológico del individuo, (en nuestro caso, el estudiante universitario) para dar cuenta de los procesos que determinan su condición de ser resiliente.

Para tal fin, asumimos las aportaciones que sustenta el modelo ecológico de resiliencia, en el que se sostiene que: “...el individuo, se halla inmerso en una ecología determinada por diferentes niveles que interactúan entre sí, ejerciendo una influencia directa en su desarrollo humano. Los niveles que conforman el marco ecológico son: el individual, el familiar, el comunitario y el cultural" (Bronfenbrenner, 1981, citado en Melillo, 2001:34)

A efectos de la investigación que hemos realizado, consideramos como objetivo de la misma el encontrar la existencia de las que podemos denominar "causas necesarias", es decir identificar los eventos negativos, que están presentes en la vida, de los estudiantes, tomando en cuenta la constelación de múltiples riesgos, en donde las complejidades del mundo real, toman forma a partir de los aspectos individuales, sociales, familiares y comunitarios que caracterizan al entorno en el que un estudiante se mueve.

Hemos dirigido nuestra observación, no a niños como ha sido habitual en las investigaciones históricamente, sino a estudiantes universitarios, como en el caso del estudio de Bermúdez, pero en nuestro estudio, de la Escuela Universitaria de Enfermería de Algeciras, perteneciente a la Universidad de Cádiz.

La consideración de dificultades o adversidades, no se ha realizado desde nuestra perspectiva, sino que han sido los propios actores, los que han 
establecido cuáles son las fuentes de adversidad real en su vida como estudiantes universitarios, partiendo de su definición de "adversidad", es decir, desde sus propios valores y creencias.

Nuestra investigación ha sido de tipo cualitativo, y hemos realizado técnicas grupales con los estudiantes de la citada escuela, estableciendo una sola diferencia, la de género para la constitución de los grupos focales en primera instancia, y obviando posteriormente esta categoría.

\section{MATERIAL Y MÉTODOS}

Siguiendo a Bericat y Beltrán, entre otros, hemos diseñado un estudio cualitativo que utiliza diferentes tipos de técnicas, ante ello debemos tener una actitud abierta tal como explicitan los autores:

"Sin duda la distinción entre las orientaciones metodológicas cuantitativa y cualitativa constituye un buen ejemplo de código binario, excluyente, que no logra reducir la realidad a sus categorías sino a costa de una insatisfactoria simplificación. La divisoria entre los métodos cualitativo y cuantitativo es la expresión bifurcada de la complejidad social. "Gracias a ella, se opera una reducción de la complejidad social que, lejos de crear situaciones simples y homogéneas, se caracteriza por crear una nueva complejidad. La operación de reducción consiste en reconducir las infinitas posibilidades que el mundo brinda a un código binario que fija sólo dos conductas posibles " (Bericat, E., 1998).

"Acerca de la antinomia cantidad-cualidad ha podido escribir Brodbeck: <<la cuantificación se ha tornado en símbolo de prestigio para muchos científicos sociales... Para otros, por el contrario la cuantificación es anatema... Tanto el sueño ilusionado como la pesadilla son reacciones desproporcionadas. La lógica de la situación no justifica ni el exceso de celo ni la repudiación total..., pues la dicotomía cantidad - cualidad es espúrea. La ciencia se refiere al mundo, esto es a las propiedades y relaciones entre las cosas..." (Beltrán, M., 1989)

“... los métodos empíricos cuantitativo y cualitativo son, cada uno de ellos, necesarios in sua esfera, in suo ordine, para dar razón de aspectos, componentes o planos específicos del objeto de conocimiento. No solo no se excluyen mutuamente, sino que se requieren y se complementan, tanto más cuanto el propósito de abarcar la totalidad del objeto sea más decidido.” (Beltrán, M.,1989).

El estudio, pues, ha sido cualitativo, para nosotros la "realidad" que pretendemos conocer es cognoscible a través de la vida cotidiana de los actores sociales, pues ellos crean esa realidad, conocen las dificultades, y son capaces de transmitirlo a los investigadores.

Nosotros formulamos nuestra perspectiva investigadora de la siguiente forma: ontológicamente, desde una perspectiva hermenéutica cuyo foco central de análisis, comprende a la perspectiva de los actores observados (alumnos); epistemológicamente: adoptaremos como marco teórico, el constructivista que mantiene una posición relativista de la realidad social, realidad que se construye sobre la base de las interacciones en la vida cotidiana; y metodológicamente, nos hemos decantado por la utilización de técnicas de búsqueda de consenso, dado que los investigadores considerábamos que los alumnos poseían la información que buscábamos, y lo que se precisaba era "extraérsela" a ellos, evitando en lo posible los sesgos individuales; es por ello que nos hemos decantado por tres técnicas de esta metodología, dos que realizaremos asociadas, una Técnica Delphi (TD) y una Técnica de Grupo Nominal (TGN); y finalmente una Técnica de Brainstorming (TB), siendo siempre los informantes alumnos.

El desarrollo de dos TGN inicialmente para que alumnos y alumnas (de forma separada) aporten sobre el concepto de "adversidad", desde la perspectiva de los estudiantes, y posteriormente dos grupos focales que aclaren en la medida de lo posible los "indicadores de adversidad" establecidos; todo ello nos permitirá contar con un marco conceptual propio a la cultura que distingue a los sujetos de estudio, para dar cuenta de los factores que pueden determinar la puesta en marcha de los procesos resilientes.

El objetivo, era comprobar como identificaban la adversidad o dificultad susceptible de producir resiliencia, el alumnado de la Escuela Universitaria de Enfermería de Algeciras (España).

La Unidad de observación como ya se mencionó es la Escuela Universitaria de Enfermería de Algeciras en España, situada en la Comarca del Campo de Gibraltar, siendo una característica 


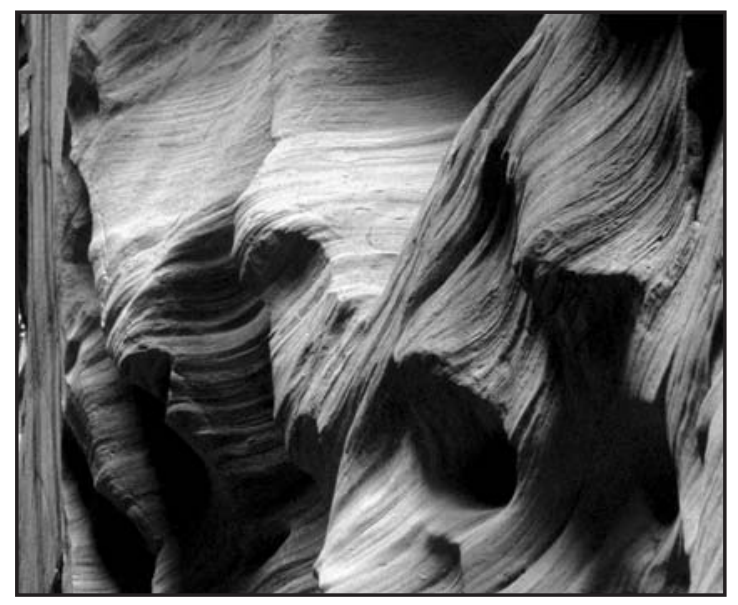

importante de la misma, el ser una zona endémica de paro tal como la provincia a la que pertenece, que es la de mayor paro de España, Cádiz. La unidad de análisis, son los alumnos de todos los cursos de la mencionada institución, y su concepción de las principales dificultades / adversidades que afectan en su formación.

Las técnicas realizadas han sido: una Técnica Delphi (Delbecq, Van de Ven et al, 1989) una técnica donde los participantes no saben quien son los otros, que se realiza mediante varios cuestionarios sucesivos, y donde se llega al consenso por votación individual de los ítems por parte de los participantes. En ella participaron 30 alumnos (diez por curso, que se presentaron voluntarios); una vez obtenidos los resultados priorizados de esta técnica, estos fueron analizados por un grupo de siete alumnos elegidos al azar entre los anteriores participantes, mediante una Técnica de Grupo Focal (Merton25,1946), lo que permitió clarificar el sentido con que habían sido aportados, ya que esta técnica a diferencia de la anterior permite la interacción comunicativa entre los participantes cara a cara; y por último, mediante un Técnica de Brainstorming, realizamos la revisión de los Ítems, mediante cinco alumnos del grupo inicial, elegidos al azar.

\section{RESULTADOS}

En el proceso de la Técnica Delphi afloraron, inicialmente, cincuenta y un problemas que afectaban a la formación de los alumnos, y eran de de todo tipo, siendo clasificados según unas categorí- as establecidas por los alumnos, en: problemas económicos, problemas personales, problemas organizacionales, problemas sociales, problemas académicos y otro tipo de problemas (Anexo $\mathrm{n}^{\circ} 1$ : Panel 1).

Como podemos observar en todas las fases, por los cuestionarios, han dominado los problemas económicos en cuanto a número, menos en la primera en la cual esta categoría además de no ser la primera tenía a otras dos con el mismo número de Ítems que ella.

Los cinco problemas priorizados por los alumnos en esta TD, fueron los siguientes y en este orden: estrés debido a la cantidad de clases y horario además de las prácticas clínicas; no tener beca; desplazamientos a casa caros; gastos en almuerzos fuera de casa; y falta de tiempo para las actividades propuestas (académico).

Estos ítems quisimos precisarlos con una TGN que nos llevó a las siguientes manifestaciones, en cuanto al significado que habían querido dar con cada Item. (Tabla $\mathrm{n}^{\circ} 2$ ):

- Estrés debido a la cantidad de clases y horario además de las prácticas clínicas, fue "traducido" de la siguiente forma por los alumnos: existencia de una mala organización docente, que impide tener tiempo real para estudiar

- No tener beca, ellos afirmaban que si no hay "beca" no se podría estudiar, esto es un problema añadido para los que vienen de "fuera de Algeciras", porque tienen mayores gastos.

- Desplazamientos a casa caros, sobre ello y relacionándolo con el Distrito único existente en Andalucía, decían: esta es una situación obligada, y que por lo tanto "cursa" con gastos

- Gastos en almuerzos fuera de casa; debido a horarios de mañana y tarde, referidos a: la organización y dedicación horaria esto implica muchos almuerzos y caros, y

- Falta de tiempo para las actividades propuestas, sobre lo que apostillaban, que: las denominadas actividades dirigidas son un añadido a las clases, y aunque los profesores parecen acotar el tiempo dedicado a ellas, los límites de dedicación se superan con creces. La dis- 
tribución de las clases y de las actividades presenciales es diferente entre cursos, también el acceso a los recursos (los informáticos, por ejemplo). Habría que cambiar no las actividades pero si ajustarlas en tiempo, a lo que se tiene.

Por último la TB, consiguió que los alumnos estuvieran en una situación de conciencia de la libertad para expresarse y a las preguntas aportadas comentaron dificultades como las que siguen:

- Problemas económicos, existe por su parte una concentración en el aspecto económico.

- Problemas personales, la falta de compañerismo dificulta el estudio, porque no se aprovechan los recursos disponibles; existe mucha competitividad, esto genera una falta de compañerismo; y falta de apoyo por parte de los alumnos a la hora de enfrentarse a los profesores.

- Problemas organizacionales, no se menciona ninguno.

- Problemas sociales, los participantes no mencionan ninguno.

- Problemas académicos, no sabes estudiar en primero; hay profesores que no saben explicar; hay clases que en vez de explicar el profesor, expone el alumno; tus fallos son castigados con humillación, debido a profesores retrógrados; se necesita que los profesores te escuchen; no hay relación alumno- profesor; no hay comunicación; hay asignaturas que no se pueden dar en un cuatrimestre; hay diferencias entre el nivel de los profesores; hay diferencias entre en nivel previo de los alumnos; hay también una mano larga con determinados alumnos; el nivel de exigencia es bajo, creo que trabajaba y se me exigía más en el bachillerato, excepto algunas asignaturas; y están de acuerdo en que los trabajos no tienen porqué suspender una asignatura o bajar su nota frente a un examen.

- Y otro tipo de problemas, donde no se mencionó ninguno.

Como vemos los problemas académicos, algunos graves, aparecen en esta técnica, trece nada menos, mientras en aspectos personales solo aparecen tres, y en económicos solo uno.

\section{CONCLUSIONES}

En un país como España situado entre los 12 países más industrializados del mundo, la plena expresión del capitalismo baña todas las esferas de la actividad cotidiana, mejor coche, mejor casa, mejor, mejor y mejor... Si bien es un país donde aspectos de la llamada sociedad del bienestar también están bien enraizados quizás con la ayuda de varios gobiernos de corte socialista, que han hecho que se siga pensando en la salud y la educación como derechos inalienables de todos los individuos que habitan en el país, tanto españoles como extranjeros.

Los estudios Universitarios, en España, si bien no son gratuitos, tienen dos particularidades: una, la matrícula que se cobra por ellos no responde al coste real de la misma, sino que el decalage es asumido por el Estado es decir por la comunidad; y dos, se establecen becas dependiendo de los ingresos, para matrícula, alojamiento, desplazamientos, etc.

Es a la luz de este contexto como podemos observar que a pesar de todo este "bienestar", en la aportación de los alumnos los problemas económicos eran mayoría y así el final de la Técnica Delphi, y ya priorizados estos ítems, parecía que iba a ser monotemático pero aparecen dos, uno en primer lugar de la priorización, que es personal y el último que es organizacional.

Es importante, pues, que sobre el amasijo inicial de problemas personales, haya restado uno solo, y que los problemas económicos se hayan concentrado en tres, y por último, el hecho de que la institución y su organización también se vean analizadas dada la importancia, que a veces se obvia, del contexto.

La Técnica de grupo focal al aclarar estos cinco Ítems, nos habla en primer lugar de que existe una mala organización docente, ya que impide tener tiempo real para estudiar, que es un problema personal, pero que se apoya en la falta de racionalidad organizativa, esto es bastante común pues como decía Goofman (1992) las instituciones llevan una dinámica propia, que es manejada o controlada por los funcionarios y no por los internos, en este caso alumnos.

La queja fundamental de no tener beca, no se sostiene cuando los padres y/o tutores poseen medios económicos para pagar esa matricula que 
es reducida, pero en el caso de Andalucía, que esta compuesta de 8 provincias con más de 7 millones de habitantes, se han creado últimamente varias universidades como una apuesta política, que para que tengan alumnos se ha definido toda la Comunidad Autónoma como si fuera un solo distrito, y los jóvenes son enviados a estudiar obligatoriamente fuera de casa muchas veces, existiendo esos estudios en su provincia, sufriendo el pecunio familiar un grave quebranto, lo cual es recogido por los alumnos.

Cierto es, que esta complicación de vivir fuera de casa se amplifica con el hecho de que la organización de la Diplomatura implica comer fuera de casa muy a menudo por la combinación con las actividades prácticas que se hacen en centros sanitarios que pueden estar hasta a 40 kilómetros.

Por último entre esas dificultades, la adaptación al Espacio Europeo de Educación Superior (en nuestra Escuela de Enfermería llevamos pilotando el nuevo sistema tres años) ha implicado convertir al profesor en un guía, para ello muchos hemos disminuido la carga teórica y hemos aumentado las actividades dirigidas, y las tutorías, pero en muchos casos las denominadas actividades dirigidas son un añadido a las clases, y los profesores no parecen acotar el tiempo dedicado a ellas, por ello los límites de dedicación se superan con creces. La distribución de las clases y de las actividades presenciales es diferente entre cursos, también el acceso a los recursos, por ejemplo, los informáticos y bibliográficos.

Habría que cambiar - de verdad- las técnicas y la dilación a las mismas, no abandonar las actividades pero si ajustarlas en tiempo al que realmente se dispone. Si bien hasta aquí todo es fácilmente explicable, los resultados de la tormenta de ideas, nos dejan un poco perplejos, dado que aquí lo central de la problemática es lo académico, parece como si pasadas las técnicas iniciales, en esta se han explayado, los alumnos- estableciendo los problemas que tienen realmente, así los problemas académicos me parecen gravísimos:

- Algunos de carácter general y previo, como el hecho de que el nivel de exigencia sea inferior al Bachillerato, o el hecho de que haya una heterogeneidad muy marcada en la formación de los alumnos.
- La crítica a los profesores es lapidaria, desde que en vez de exponer el profesor lo hace el alumno, pasando por el hecho de que hay profesores que no saben explicar (en general), que los profesores no escuchan, que no hay comunicación (alumno - profesor), que también entre los profesores los niveles son muy diferentes, hay una complacencia por parte del profesorado con ciertos alumnos que también puede ser una mano negra, y lo peor de todo es leer lo siguiente "Tus fallos son castigados con humillación, debido a profesores retrógrados", esta frase no necesita explayarse más en ella.

- No saber estudiar en primer curso de la Universidad, después de 15 años estudiando es grave.

En cuanto problemas más bien personales o también se les podría haber clasificado como sociales, encontramos la falta de compañerismo por la mucha competitividad, y lo que a veces produce la misma, y es que no se aprovechan bien los recursos (libros,...); por último en estas carreras e instituciones donde el contacto es tan cercano, se confunden muchas veces las distancias, los roles,... los espacios personales. La falta de apoyo al delegado, o de un frente común ante problemas genéricos. En resumen hay problemas económicos, pero los problemas fundamentales son académicos.

\section{BIBLIOGRAFÍA}

- Bermúdez, M. T. (2009) "Etnografía de la Resiliencia en Educación Superior" Tesis doctoral leída en la Universidad Autónoma del Estado de Hidalgo. Pachuca. Pág. 115 (Tesis doctoral)

- Beltrán, M. (1991) “La realidad social”. Madrid. Ed. Tecnos. Pág. 39.

- Beltrán, M. (1991) “La realidad .”op. cit, Pág. 40.

- Bericat, E. (1998) La integración de los métodos cuantitativo y cualitativo en la investigación social. Barcelona: Ariel Sociología. Pág, 12.

- Cyrulnik, B. (2002) Los patitos feos. La resiliencia: una infancia infeliz no determina la vida. Barcelona: Gedisa.

- Delbecq, A. L.; Van de Ven, A. H.; Y Gustaffson, D. H. (1989) “Técnicas grupales para la planeación”. Ed. Trillas. México.

- Goffman, E. (1992) Internados. Buenos Aires. Ed Amorrortu. - Infante, F. (2001) La Resiliencia como proceso: una revisión de la literatura reciente. En Resiliencia descubriendo las propias fortalezas. Buenos Aires, Barcelona: Paidós. Pp. 32-53. Kobasa, S. C., Maddi, S. R. (1977) "Existential personality theory" en Corsini, R. "Current personality theory". Itasca, III. Peacock. 
- Kobasa, S. C., Maddi, S. R., Kahn, S. (1982) "Hardiness and Health. A prospective study". Journal of Personality and Social Behavior", 22: 368-378.

- Kobasa, S. C., Maddi, S. R., Kahn, S. (1993) "Hardiness and Health. A prospective study. Clarification". Journal of Personality and Social Behavior, 65 (1) 207.

- Luthar S. S. "Annotation: Methodological and conceptual issues in the study of Resilience". Journal of Child Psychology and Psychiatry. 1993; 34: 441-453.

- Malinowski, B. (1973) "A scientific theory of culture and o enther essay" North Carolina, University Press.

- Manciaux, M. ; Vandistendael, S.; Lecomte, J.; y Cyrrulnik, B. (2003) La resiliencia: Estado de la cuestión. En la resiliencia: resistir y rehacerse. Barcelona: Gedisa. Pp 17-27.

- Melillo, A. (2001) Prefacio. Resiliencia descubriendo las propias fortalezas. Buenos Aires, Barcelona: Paidos. Pp. 17-18.

- Merton, R. K.; Kendall, P. L.(1946) 'The Focused Interview', American Journal of Sociology 51: 541-557. New Cork.

- Rutter, M. (1985) "Resilience in the face of adversity: Protective factors and resistance to psychiatric disorder. British Journal of Psychiatry, 147: 598- 611.

- Rutter, M. (1993). "Resilience: Some conceptual considerations". Journal of Adolescent Health. 14: 626-631.
- Saavedra, G. E. (2002) El enfoque cognitivo procesal sistémico, como mirada para la comprensión de la resiliencia. Tesis Doctoral 2001-2002. Universidad Católica de Maule. Pág. 5 (Tesis doctoral).

- Suárez, O. N. (2001) Una concepción latinoamericana de Resiliencia. En Resiliencia descubriendo las propias fortalezas. Buenos Aires, Barcelona: Paidos. (Pp. 67-82).

- Suárez, O. N. (2004) Introducción. En Resiliencia y subjetividad: Los ciclos de la vida. Argentina. Paidós. Pp. 17-20.

- Theis, A. (2003) La resiliencia en la literatura científica, en Manciaux, La resiliencia: resistir y rehacerse. Barcelona: Gedisa. Pp. 45-59.

- Tylor, E. B. (1924) "Primitive culture.Researches in the development of mythology, philosophy, religion, language, art and customs" 2 vols. 7th ed. New York: Brentano's.

- Vandistendael, S. (2003) La resiliencia en lo cotidiano. Barcelona. Gedisa.

- Suárez, O. N. (2001) Una concepción latinoamericana de Resiliencia. En Resiliencia descubriendo las propias fortalezas. Buenos Aires, Barcelona: Paidos. Pp. 67-82.

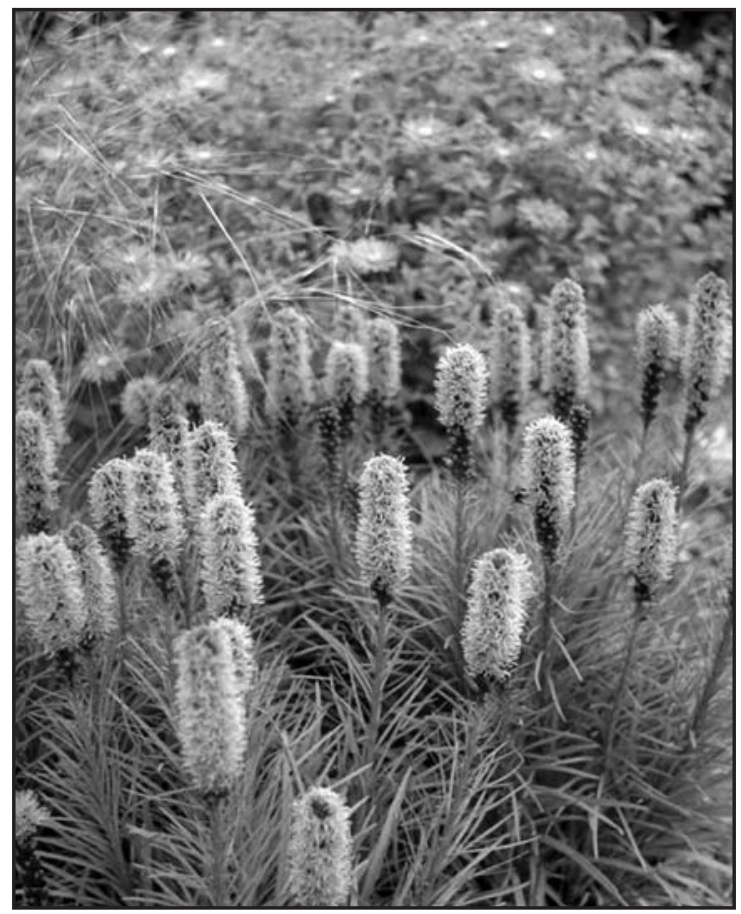


Apreciado alumno.

\section{CARTA 1}

Ante todo agradecerte tu participación en este estudio sobre la resiliencia en los estudiantes de la Escuela Universitaria de Enfermería de Algeciras.

En principio la resiliencia la vamos a considerar como la capacidad de ser humano para enfrentar la adversidad, superarla y ser fortalecido o transformado positivamente.

La resiliencia ha sido estudiada generalmente en niños, nosotros pretendemos hacerlo en universitarios, de ahí que os pidamos vuestra colaboración.

La Técnica como ya sabéis consiste en daros unos cuestionarios que debéis cumplimentar (sin comentar con los compañeros), una vez realizados lo podéis dejar en el casillero mío dentro del sobre que os pongo acompañando al cuestionario, que es anónimo y por tanto nadie sabrá aquello que contestéis.

Par contestar a este, debéis indicar cuáles son las situaciones adversas que obstaculizan el curso de vuestros estudios profesionales (sean personales, sociales, económicas...), y el plazo para devolverlo podemos contemplar el período que va desde la fecha de hoy hasta al inicio de las vacaciones de Navidad.

En Algeciras a 2 de Diciembre del 2007.

Fdo./ Dr. Alvaro Bernalte Benazet 


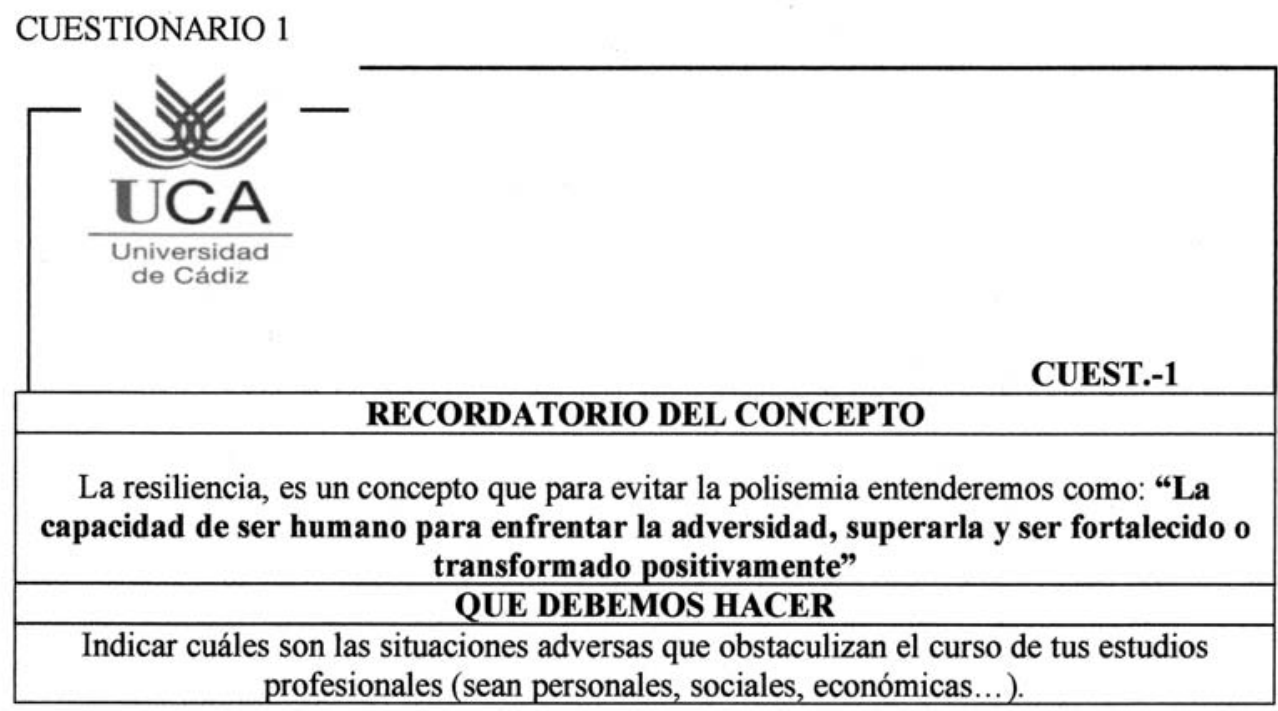

\begin{tabular}{|r|l|}
\hline 1 & \\
\hline 2 & \\
\hline 3 & \\
\hline 5 & \\
\hline 6 & \\
\hline 7 & \\
\hline 9 & \\
\hline 10 & \\
\hline 11 & \\
\hline 12 & \\
\hline & \\
\hline & \\
\hline & \\
\hline
\end{tabular}


PANEL - 1 RESPUESTAS AL PRIMER CUESTIONARIO

\section{Situaciones adversas que obstaculizan el curso de tus estudios profesionales}

\begin{tabular}{|l|l|l|l|}
\hline \multicolumn{3}{|c|}{ Problemas económicos } & \\
\hline Mucho dinero en fotocopias & & & \\
\hline Mucho dinero en material & & & \\
\hline Muchos gastos & & & \\
\hline Gastos en almuerzos fuera de casa & & & \\
\hline No tener beca & & & \\
\hline No poder pagar la matrícula & & & \\
\hline Beca insuficiente & & & \\
\hline Desplazamientos a casa caros & & & \\
\hline Falta de ordenador & & & \\
\hline $\begin{array}{l}\text { Pocas ayudas para las familias de clase } \\
\text { media }\end{array}$ & & & \\
\hline & & & \\
\hline $\begin{array}{l}\text { Falta de tiempo para las actividades } \\
\text { propuestas }\end{array}$ & & & \\
\hline Falta de compañerismo en la clase & & & \\
\hline Carga familiar & & & \\
\hline Bajo nivel de estudios & & & \\
\hline Falta de concentración & & & \\
\hline Falta de organización & & & \\
\hline Falta de planificación & & & \\
\hline Falta de síntesis & & & \\
\hline Nivel de exigencia elevado & & & \\
\hline No satisfacción con los estudios & & & \\
\hline Motivación de la rivalidad entre compañeros & & & \\
\hline No disponer de internet & & & \\
\hline $\begin{array}{l}\text { Dificultad para reconocer las limitaciones y } \\
\text { superarlas }\end{array}$ & & & \\
\hline Esperar mucho de los estudiantes & & \\
\hline La distancia de mi casa a Algeciras & & & \\
\hline $\begin{array}{l}\text { Estrés debido a la cantidad de clases y } \\
\text { horario además de las prácticas clínicas }\end{array}$ & & & \\
\hline No me puedo permitir caprichos & & \\
\hline
\end{tabular}




\begin{tabular}{|l|l|l|l|}
\hline Gastar mucho tiempo en ocio & \multicolumn{2}{|l|}{} & \\
\hline \multicolumn{2}{|c|}{ Problemas sociales } & \\
\hline $\begin{array}{l}\text { Falta de transporte público para las clases y } \\
\text { las prácticas }\end{array}$ & & & \\
\hline No disponer de habitación para estudiar & & & \\
\hline Vivir lejos del centro & & & \\
\hline $\begin{array}{l}\text { No encontratr vivienda cerca de la } \\
\text { universidad }\end{array}$ & & & \\
\hline Material escaso para las prácticas & & & \\
\hline Celos y envidias en la clase & & & \\
\hline Problemas con mis amigos & & & \\
\hline No tener cerca los amigos & & & \\
\hline Convivir con personas desconocidas & & & \\
\hline $\begin{array}{l}\text { Difícil integrarse por que la gente es } \\
\text { diferente }\end{array}$ & & & \\
\hline No poder ver a mi familia lo que quisiera & & & \\
\hline & & & \\
\hline $\begin{array}{l}\text { Escaso margen de tiempo de entrega de las } \\
\text { actividades }\end{array}$ & & & \\
\hline Muchas actividades a través de internet & & & \\
\hline Plan de estudios limita el tiempo & & & \\
\hline Pocos recursos del aula virtual & & & \\
\hline Poco interés que despiertan los profesores & & & \\
\hline Dificultad de las asignaturas & & & \\
\hline Poco interés por las asignaturas & & & \\
\hline $\begin{array}{l}\text { No saber coger apuntes en clase y la carga } \\
\text { que ello comporta }\end{array}$ & & & \\
\hline Pocos lugares de estudio & & \\
\hline El proyecto piloto & & \\
\hline & & & \\
\hline Los ruidos de la calle me impiden estudiar & & & \\
\hline $\begin{array}{l}\text { Poca distribución de la información } \\
\text { universitaria }\end{array}$ & & & \\
\hline & & & \\
\hline
\end{tabular}


Anexo 2. Segunda fase de la Técnica Delphi (Carta 2, Cuestionario 2 y panel 2)

\section{CARTA-2}

Apreciado alumno.

Ante todo agradecerte tu participación en este estudio sobre la resiliencia en los estudiantes de la Escuela Universitaria de Enfermería de Algeciras.

Recordaros que la resiliencia la vamos a considerar como la capacidad de ser humano para enfrentar la adversidad, superarla y ser fortalecido o transformado positivamente; debemos comentar, así mismo, que la resiliencia ha sido estudiada generalmente en niños, nosotros pretendemos hacerlo en universitarios, de ahí que os pidamos vuestra colaboración.

En este segundo cuestionario, nos tenéis que indicar de entre vuestras respuestas al anterior tres cosas:

a) Cuales son las 10 respuestas más importantes, poniendo en el casillero de importancia el 1 a la más importante y el 10 a la menor.

b) Encontrareis una casilla una para indicar si estáis de acuerdo con el item, si es así colocad un $\mathrm{X}$ en la casilla

c) Encontrareis una casilla para indicar cuando NO estáis de acuerdo, si es así colocad un X en la casilla

Cerrad el sobre y dejadlo en el casillero, dado que nos hemos retrasado un poquillo, os rogariamos nos contestéis antes de 10 días. 


\section{CUESTIONARIO 2}

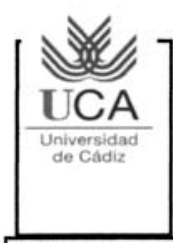

CUEST-2

La resiliencia, es un concepto que para evitar la polisemia entenderemos como: "La capacidad de ser humano para enfrentar la adversidad, superarla y ser fortalecido o transformado positivamente"

\begin{tabular}{|c|c|c|c|c|}
\hline $\mathrm{N}^{\circ}$ & items & PUNTUACIÓN & DE ACUERDO & \begin{tabular}{|l} 
EN \\
DESACUERDO \\
\end{tabular} \\
\hline 1 & Mucho dinero en fotocopias & & & \\
\hline 2 & Mucho dinero en material & & & \\
\hline 3 & Muchos gastos & & & \\
\hline 4 & Gastos en almuerzos fuera de casa & & & \\
\hline 5 & No tener beca & & & \\
\hline 6 & No poder pagar la matrícula & & & \\
\hline 7 & Beca insuficiente & & & \\
\hline 8 & Desplazamientos a casa caros & & & \\
\hline 9 & Falta de ordenador & & & \\
\hline 10 & Pocas ayudas para las familias de clase media & & & \\
\hline 11 & Falta de tiempo para las actividades propuestas & & & \\
\hline 12 & Falta de compañerismo en la clase & & & \\
\hline 13 & Carga familiar & & & \\
\hline 14 & Bajo nivel de estudios & & & \\
\hline 15 & Falta de concentración & & & \\
\hline 16 & Falta de organización & & & \\
\hline 17 & Falta de planificación & & & \\
\hline 18 & Falta de síntesis & & & \\
\hline 19 & Nivel de exigencia elevado & & & \\
\hline 20 & No satisfacción con los estudios & & & \\
\hline 21 & Motivación de la rivalidad entre compañeros & & & \\
\hline 22 & No disponer de internet & & & \\
\hline 23 & Dificultad para reconocer las limitaciones y superarlas & & & \\
\hline 24 & Esperar mucho de los estudiantes & & & \\
\hline 25 & La distancia de mi casa a Algeciras & & & \\
\hline
\end{tabular}




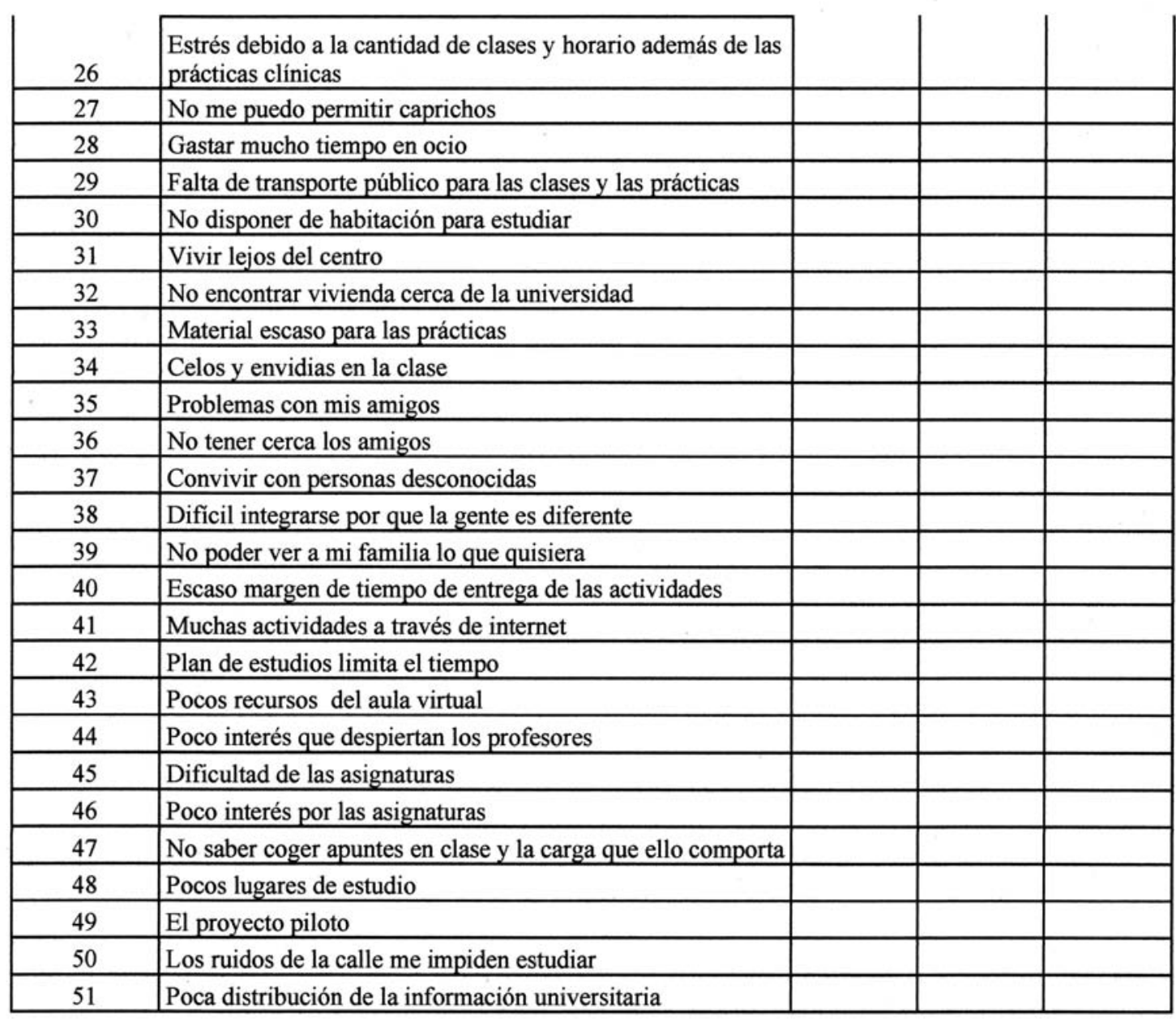




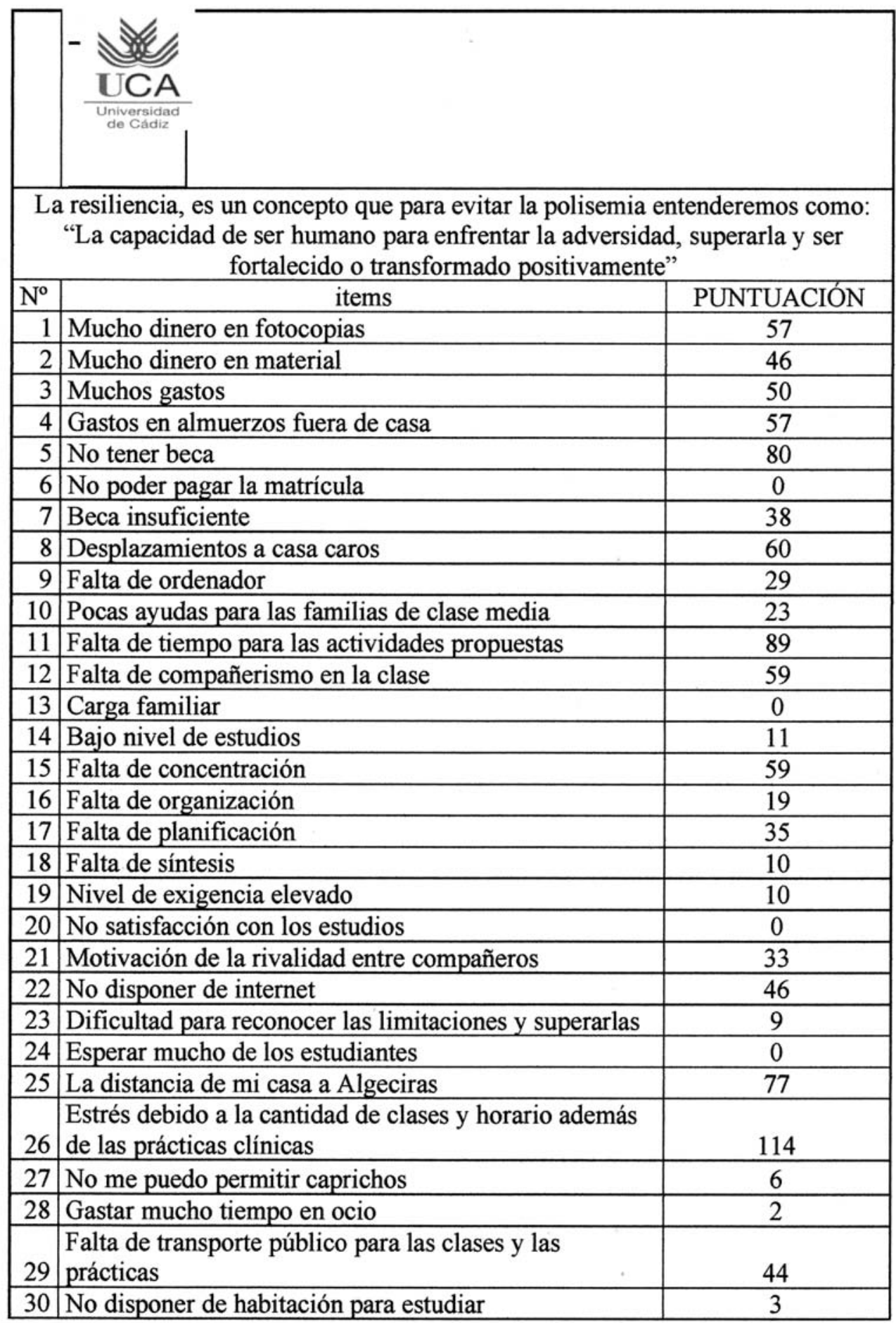




\begin{tabular}{|c|l|c|}
\cline { 2 - 2 } 31 & Vivir lejos del centro & 10 \\
\hline 32 & No encontrar vivienda cerca de la universidad & 16 \\
\hline 33 & Material escaso para las prácticas & 20 \\
\hline 34 & Celos y envidias en la clase & 29 \\
\hline 35 & Problemas con mis amigos & 4 \\
\hline 36 & No tener cerca los amigos & 31 \\
\hline 37 & Convivir con personas desconocidas & 13 \\
\hline 38 & Difícil integrarse por que la gente es diferente & 9 \\
\hline 39 & No poder ver a mi familia lo que quisiera & 59 \\
\hline 40 & Escaso margen de tiempo de entrega de las actividades & 13 \\
\hline 41 & Muchas actividades a través de internet & 48 \\
\hline 42 & Plan de estudios limita el tiempo & 28 \\
\hline 43 & Pocos recursos del aula virtual & 0 \\
\hline 44 & Poco interés que despiertan los profesores & 5 \\
\hline 45 & Dificultad de las asignaturas & 19 \\
\hline 46 & Poco interés por las asignaturas & 16 \\
\hline & No saber coger apuntes en clase y la carga que ello & \\
47 & comporta & 12 \\
\hline 48 & Pocos lugares de estudio & 11 \\
\hline 49 & El proyecto piloto & 1 \\
\hline 50 & Los ruidos de la calle me impiden estudiar & 6 \\
\hline 51 & Poca distribución de la información universitaria & 11 \\
\hline
\end{tabular}


Apreciado alumno.

Ante todo agradecerte tu participación en este estudio sobre la resiliencia en los estudiantes de la Escuela Universitaria de Enfermería de Algeciras.

Recordaros que la resiliencia la vamos a considerar como la capacidad de ser humano para enfrentar la adversidad, superarla y ser fortalecido o transformado positivamente; debemos comentar, así mismo, que la resiliencia ha sido estudiada generalmente en niños, nosotros pretendemos hacerlo en universitarios, de ahí que os pidamos vuestra colaboración.

En este tercer cuestionario, nos tenéis que indicar de entre vuestras respuestas al anterior cuales son las 5 respuestas más importantes, poniendo en el casillero de importancia el 1 a la más importante y el 5 a la menor.

En Algeciras a 5 de Febrero del 2008.

Fdo./ Dr. Alvaro Bernalte Benazet 


\section{CUESTIONARIO 3}

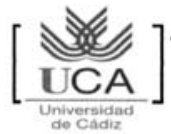

CUESTIONARIO - 3

\begin{tabular}{|c|c|c|}
\hline \multicolumn{3}{|c|}{ RESULTADOS DE LA TÉCNICA DELPHI } \\
\hline $\mathrm{N}^{\circ}$ & items & PUNTUACIÓN \\
\hline 1 & $\begin{array}{l}\text { Estrés debido a la cantidad de clases y } \\
\text { horario además de las prácticas clínicas }\end{array}$ & \\
\hline 2 & No tener beca & \\
\hline 3 & Desplazamientos a casa caros & \\
\hline 4 & Gastos en almuerzos fuera de casa & \\
\hline 5 & $\begin{array}{l}\text { Falta de tiempo para las actividades } \\
\text { propuestas }\end{array}$ & \\
\hline 6 & Mucho dinero en fotocopias & \\
\hline 7 & La distancia de mi casa a Algeciras & \\
\hline 8 & Falta de compañerismo en la clase & \\
\hline 9 & No poder ver a mi familia lo que quisiera & \\
\hline 10 & Falta de concentración & \\
\hline
\end{tabular}

\section{PANEL - 3 RESPUESTAS AL CUESTIONARIO 3}

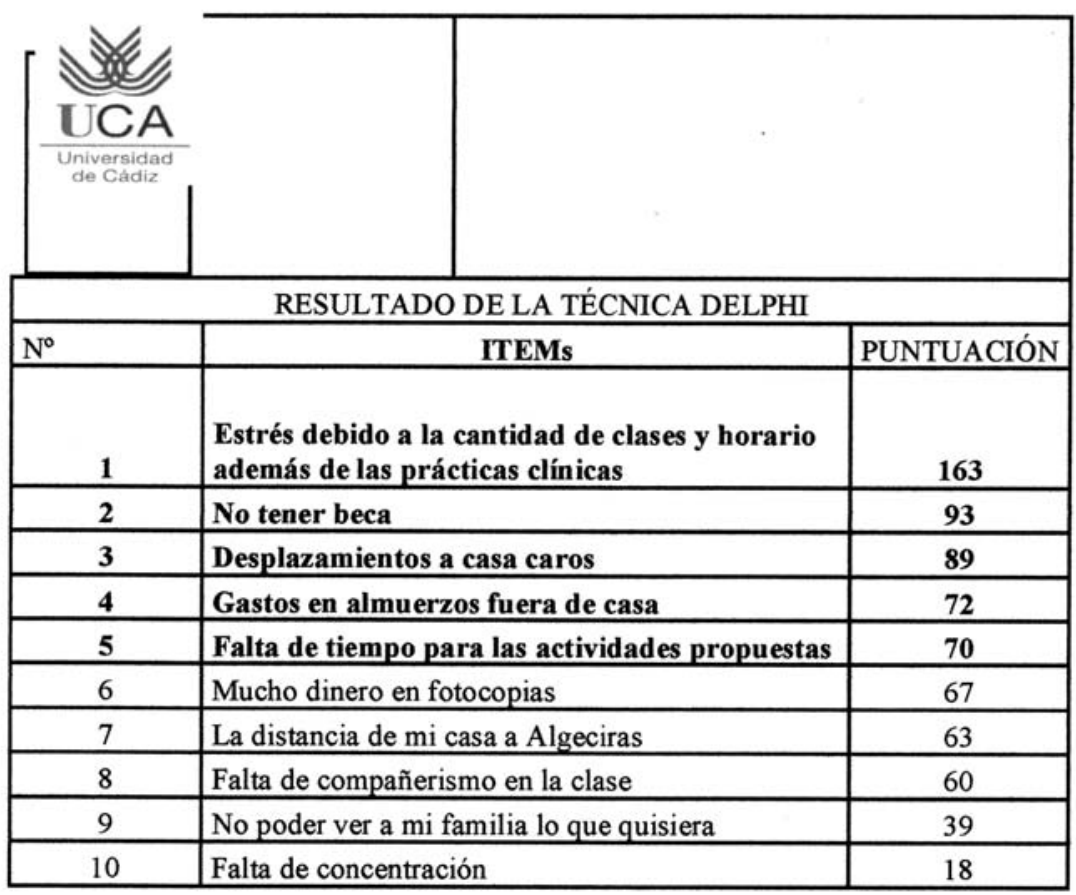




\section{Anexo 4. Tabla con los resultados del Grupo focal RESULTADOS GRUPO FOCAL. Consenso sobre el significado de los ITEMS}

\section{1.- ¿Qué entienden por estrés debido a la cantidad de clases y horario además de las prácticas clíni- cas?}

Que existe una mala organización docente (clases, prácticas, actividades dirigidas...) ya que impide tener tiempo real para estudiar

Observación: Se habló relajadamente, los argumentos de los alumnos se apoyan unos en otros; se enfatiza con el lenguaje no verbal.

Contradicciones: No observamos contradicciones

En las aportaciones se dieron algunas soluciones a esto, así: unos decían que no hubieran clases y prácticas en el mismo período, otros hablaban de un cierto equilibrio necesario, no podía salir de las tres de otro pueblo y estar en Algeciras a las cuatro.

\section{2.- ¿Consideran el no tener beca un factor adverso, por qué? (todos deben tener beca)}

Si no hay "beca" no se podría estudiar, esto es un problema añadido para los que vienen de "fuera", porque tienen mayores gastos.

Observación: Se habla relajadamente y los miembros del grupo se apoyan en sus intervenciones; en cada intervención se apoyan con lenguaje no verbal.

Contradicciones: No observamos contradicciones

\section{3.- ¿Qué implica el que ir a casa sea caro? (lejanía de la familia...?)}

Esta es una situación obligada, y que por lo tanto "cursa" con gastos

Observación: Hay disparidad ya que mientras unos consideran la obligación, otros consideran que esto es igual porque está ligado al hecho de querer estudiar

\section{Contradicciones: No observamos contradicciones}

Se comenta el síndrome del nido vacío, que parece afectar a los jóvenes alejados de su casa, la comida de las madres y ... el salir con los amigos; se insiste en los precios para estudiante no son tales, y en el hecho de que estar lejos de casa aumenta los gastos.

\section{4.- ¿Los gastos en almuerzos fuera de casa son un problema, por qué?}

Dada la organización y dedicación horaria esto implica muchos almuerzos y caros

Observación: De nuevo un problema viene abonado por la mala organización, hay una concentración de las asignaturas optativas por la tarde. Todos de acuerdoy pendientes de las conclusiones.

Contradicciones: No han existido.

\section{5.- ¿Esta falta de tiempo para las actividades que se les proponen, cómo pueden explicarlo?}

Observación: La denominadas actividades dirigidas son un añadido a las clases, y aunque los profesores parecen acotar el tiempo dedicado a ellas, los límites de dedicación se superan con creces. La distribución de las clases y de las actividades presenciales es diferente entre cursos, también el acceso a los recursos (los informáticos, por ejemplo). Habría que cambiar no las actividades pero si ajustarlas en tiempo a lo que se tiene.

Contradicciones: No las ha habido 


\section{Anexo 5. APORTACIONES A LA TÉCNICA DE BRAIMSTORMING. APORTACIONES A LA T. B.}

- Problemas económicos, existe por su parte una concentración en el aspecto económico

- Problemas personales, la falta de compañerismo dificulta el estudio, porque no se aprovechan los recursos disponibles; existe mucha competitividad, esto genera una falta de compañerismo; y falta de apoyo por parte de los alumnos a la hora de enfrentarse a los profesores.

- Problemas organizacionales, no se menciona ninguno

- Problemas sociales, los participantes no mencionan ninguno

- Problemas académicos, no sabes estudiar en primero; hay profesores que no saben explicar; hay clases que en vez de explicar el profesor, expone el alumno; tus fallos son castigados con humillación, debido a profesores retrógrados; se necesita que los profesores te escuchen; no hay relación alumnoprofesor; no hay comunicación; hay asignaturas que no se pueden dar en un cuatrimestre; hay diferencias entre el nivel de los profesores; hay diferencias entre en nivel previo de los alumnos; hay también una mano larga con determinados alumnos; el nivel de exigencia es bajo, creo que trabajaba y se me exigía más en el bachillerato, excepto algunas asignaturas; y están de acuerdo en que los trabajos no tienen porqué suspender una asignatura o bajar su nota frente a un examen

- Y otro tipo de problemas, donde no se mencionó ninguno

Como vemos los problemas académicos, algunos graves, aparecen en esta técnica, trece nada menos, mientras en aspectos personales solo aparecen tres, y en económicos solo uno.

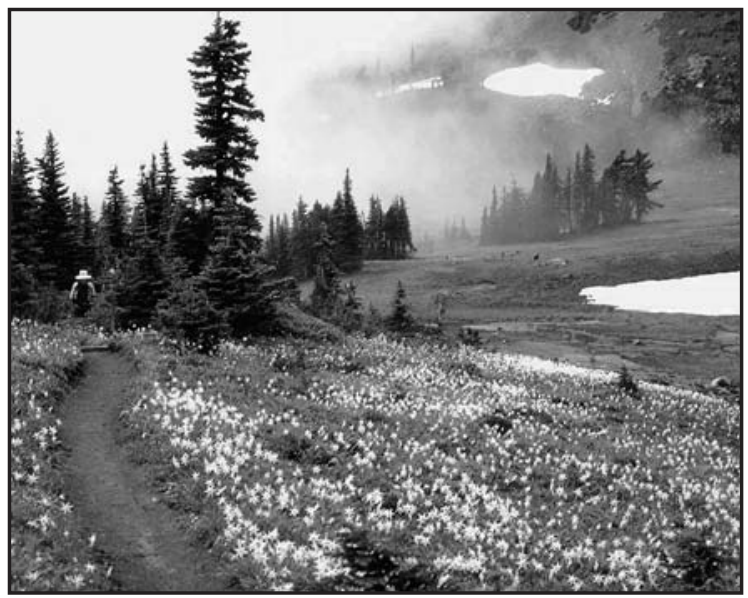




\section{Anexo 6. Aportaciones a posteriori de la T.B \\ APORTACIONES FUERA DEL CONTEXTO DE LA T. B}

$1^{\text {a }}$ PREGUNTA: Llamada de atención sobre los test.

Opiniones:

- Concentración en el aspecto económico

- No sabes estudiar en primero

- Hay profesores que no saben explicar

- Hay clases que en vez de explicar el profesor, expone el alumno

- Tus fallos son castigados con humillación, debido a profesores retrógrados

2a PREGUNTA: ¿Habéis pensado en intentar cambiar algo?

Opiniones:

- Se necesita que los profesores te escuchen.

- Falta de apoyo por parte de los alumnos a la hora de enfrentarse a los profesores.

$3^{\text {a }}$ PREGUNTA: ¿Las diferencias entre los profesores influyen en vuestro estudio?

Opiniones:

- No hay relación alumno-profesor.

- No hay comunicación

- Hay asignaturas que no se pueden dar en un cuatrimestre.

- Hay diferencias entre el nivel de los profesores.

- Hay diferencias entre en nivel previo de los alumnos.

- Hay mano larga con determinados alumnos

4 a PREGUTA: Uno de los ítems mencionados por vosotros, fue la falta de compañerismo, ¿a qué os referíais?

Opiniones:

- Existe mucha competitividad, esto genera una falta de compañerismo

- La falta de compañerismo dificulta el estudio, porque no se aprovechan los recursos disponibles.

5 PREGUNTA: ¿Creéis que se os exige demasiado nivel?

Opiniones:

- La exigencia es alta, pero no considero que por ello van a salir suficientemente preparado

- Yo si me considero suficientemente preparado, incluso si saliera ahora.

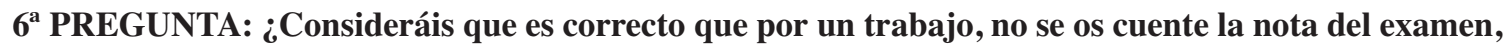
e incluso estéis suspensos?

Opiniones:

- Están de acuerdo en que los trabajos no tienen porqué suspender una asignatura o bajar su nota frente a un examen. 